\title{
HP-Eradikation schützt vor Rezidivblutungen unter ASS
}

\author{
Es wird empfohlen, Patienten unter \\ ASS-Dauertherapie mit Risikofak- \\ toren für ein peptisches Ulkus auf \\ Helicobacter pylori (HP) zu testen \\ und bei Keimnachweis eine Eradika- \\ tion durchzuführen. Es ist allerdings \\ unklar, ob diese Strategie langfristig \\ auch wirklich effektiv ist.
}

— In einer prospektiven Kohortenstudie wurden drei Gruppen von ASS-Konsumenten langfristig nachbeobachtet:

(1) ASS-Konsumenten mit Ulkusblutung und HP-Eradikation $(\mathrm{n}=249)$.

(2) HP-negative Patienten mit Ulkusblutung unter ASS $(\mathrm{n}=118)$. Nach der Ulkusheilung wurde mit enteic coated ASS weiterbehandelt.

3 Kontrollgruppe: Patienten mit durchschnittlichem Risiko für ein peptisches Ulkus, bei denen eine ASS-Therapie neu begonnen wurde $(\mathrm{n}=537)$.

In keiner Gruppe wurde ein Magenschutz verordnet. Der primäre End-

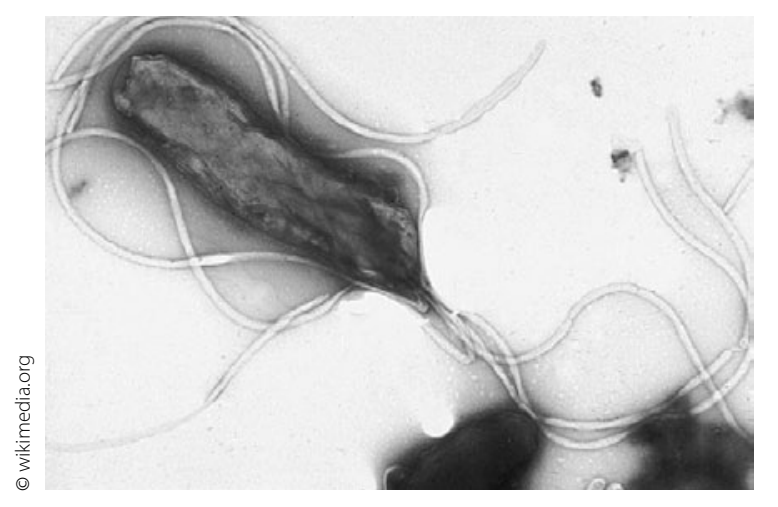

Was tun, wenn der Patient eine ASS-Dauertherapie braucht und mit $\mathrm{H}$. pylori infiziert ist? punkt war die Ulkusblutung. Das Follow-up umfasste 5048 Patientenjahre.

Die Inzidenz einer Ulkusblutung $(0,97 ; 95 \%$-Konfidenzintervall [KI] $0,53-1,80)$ unterschied sich nicht signifikant von derjenigen in der Kontrollgruppe $(0,66$; $95 \%$-KI 0,38-0,99). Die primär HP-negativen Patienten hatten ein deutlich höheres Blutungsrisiko (5,22; 95\%-KI 3,04-8,96). Das langfristige Risiko einer erneuten Ulkusblutung unter niedrigdosiertem ASS war niedrig, wenn eine HP-Infektion vorlag und diese erfolgreich behandelt wurde. HP-negative Patienten, die eine Ulkusblutung erleiden, haben dagegen ein hohes Rezidivblutungsrisiko und bedürfen einer gastroprotektiven Therapie.

- F. K. L. Chan et al.

Effects of Helicobacter pylori infection on longterm risk of peptic ulcer bleeding in low-dose Aspirin users. Gastroenterology 144 (2013) 528-535

\section{Kommentar}

Bei NSAR-Konsumenten steigert HP das Ulkusrisiko um den Faktor 1,8. Eine HP-Eradikation reduziert das Ulkusrisiko nur in der Primärprävention. Hatte der Patient schon einmal ein Ulkus unter NSAR, ist kein präventiver Effekt von einer Sanierung der Infektion zu erwarten.

Die Datenlage zur Interaktion von ASS und $\mathrm{HP}$ ist deutlich dünner und schwierig zu interpretieren. Eine kontrollierte Studie mit sechs Monaten Follow-up signalisierte einen ähnlichen präventiven Effekt der Eradikation im Hinblick auf die Prophylaxe einer Rezidivblutung unter niedrigdosiertem ASS wie $20 \mathrm{mg}$ Omeprazol. Eine weitere kontrollierte Studie mit Follow-up über zwölf Monate zeigte, dass die zusätzliche Gabe eines PPI nach HP-Eradikation einen besseren Schutz darstellt als die alleinige antibakterielle Therapie. Basierend auf dieser kontroversen Datenlage gingen die bisherigen Empfehlungen zu einer "Hosenträger-und-Gürtel"-Therapie in dieser Situation: HP-Eradikation gefolgt von einer PPI-Dauertherapie. Auf der Basis der hier vorgelegten großen Kohortenstudie ist davon auszugehen, dass eine alleinige HPEradikation einen wirksamen Schutz bietet, der sich durch die zusätzliche Gabe eines PPI vermutlich kaum verbessern lässt. Allerdings reicht die Evidenz (noch) nicht aus, die aktuell gültigen Leitlinien neu zu schreiben.

J. LABENZ = 